\title{
The apelinergic system in the developing lung: Expression and signaling ${ }^{\text {is }}$
}

\author{
Paulina Piairo $^{\mathrm{a}, \mathrm{b}}$, Rute S. Moura ${ }^{\mathrm{a}, \mathrm{b}}$, Cristina Nogueira-Silva ${ }^{\mathrm{a}, \mathrm{b}, \mathrm{c}}$, Jorge Correia-Pinto ${ }^{\mathrm{a}, \mathrm{b}, \mathrm{d}, *}$ \\ ${ }^{a}$ Life and Health Sciences Research Institute (ICVS), School of Health Sciences, University of Minho, Braga, Portugal \\ b ICVS/3B's - PT Government Associate Laboratory, Braga/Guimarães, Portugal \\ ${ }^{c}$ Department of Obstetrics and Gynecology, Hospital of Braga, Portugal \\ d Department of Pediatric Surgery, Hospital of Braga, Portugal
}

\section{A R T I C L E I N F O}

\section{Article history:}

Received 22 August 2011

Received in revised form 4 October 2011

Accepted 5 October 2011

Available online 12 October 2011

\section{Keywords:}

Apelin

APJ

Lung development

MAP kinases

\begin{abstract}
A B S T R A C T
Apelin and its receptor APJ constitute a signaling pathway best recognized as an important regulator of cardiovascular homeostasis. This multifunctional peptidergic system is currently being described to be involved in embryonic events which extend into vascular, ocular and heart development. Additionally, it is highly expressed in pulmonary tissue. Therefore, the aim of this study was to investigate the role of apelinergic system during fetal lung development. Immunohistochemistry and Western blot analysis were used to characterize apelin and APJ expression levels and cellular localization in normal fetal rat lungs, at five different gestational ages as well as in the adult. Fetal rat lung explants were cultured in vitro with increasing doses of apelin. Treated lung explants were morphometrically analyzed and assessed for MAPK signaling modifications. Both components of the apelinergic system are constitutively expressed in the developing lung, with APJ exhibiting monomeric, dimeric and oligomeric forms in the pulmonary tissue. Pulmonary epithelium also displayed constitutive nuclear localization of the receptor. Fetal apelin expression is higher than adult expression. Apelin supplementation inhibitory effect on branching morphogenesis was associated with a dose dependent decrease in p38 and JNK phosphorylation. The results presented provide the first evidence of the presence of an apelinergic system operating in the developing lung. Our findings also suggest that apelin inhibits fetal lung growth by suppressing p 38 and JNK signaling pathways.
\end{abstract}

(c) 2011 Elsevier Inc. All rights reserved.

\section{Introduction}

The existence of the apelinergic system began to unfold in 1998 when APJ (angiotensin II receptor-like 1), a formerly orphan Gprotein coupled receptor (GPCR), and its ligand were finally paired by Tatemoto et al. [45]. Apelin was identified as an endogenous ligand for APJ receptor. This peptide is translated as a 77-amino acid precursor, which undergoes proteolytic maturation generating shorter active apelin peptides. Apelin-36 was the first of these shorter C-terminal sequences being described to bind and activate APJ [45]. Also Apelin-17, Apelin-13 and its pyroglutamyl isoform which is resistant to degradation, (Pyr1)-Apelin-13, were proven to act as functional ligands of APJ and in some cases exhibited much higher biological activity than Apelin-36 [45,17,18,25,46].

\footnotetext{
is Grants: This project was funded by Fundação para a Ciência e a Tecnologia (PTDC/SAU-OBD/108051/2008), PP was supported by Fundação para a Ciência e a Tecnologia (reference SFRH/BD/33410/2008). RSM was supported by Fundação para a Ciência e a Tecnologia (reference SFRH/BPD/15408/2005).

* Corresponding author at: Escola de Ciências da Saúde, Universidade do Minho, Campus de Gualtar, 4710-057 Braga, Portugal. Tel.: +351 253604 910; fax: +351253604862.

E-mail address: jcp@ecsaude.uminho.pt (J. Correia-Pinto).
}

Apelin receptor was originally identified as a receptor related to angiotensin II receptor 1, due to high homology between the two receptor proteins [38]. This receptor is 380 amino acids long, consists of seven transmembrane domains and also includes a signal sequence that allows agonist-independent nuclear localization, a feature that may be cell-specific [27]. GPCRs are the estimated targets of nearly half of all currently available clinically used drugs [11] and are key components of the signal transduction machinery [35]. Binding of apelin to APJ activates second messenger signaling cascades after coupling to $G$ proteins, which results in activation of central signaling molecules such as mitogen-activated protein kinases (MAPKs) and the PI3K/AKT pathway that are responsible to instigate multiple biological responses [1,28-31].

The apelinergic system has a widespread pattern of distribution in human and animal tissues and its established physiological actions are extensive. Overall, apelin and APJ mRNA transcripts and respective peptides, are abundantly present in central nervous system and also in peripheral tissues such as vascular endothelium, heart, lung, kidney and mammary gland [18,25,40,32,10,21], suggesting a functional role of apelin/APJ in these tissues. Remarkably the bulk of the studies report cardiovascular actions of apelin/APJ. Moreover, this peptidergic system has been proposed to have a role in body fluid homeostasis, immunologic modulation, diabetes and 
obesity. [39,12,26,22,4]. Recently the apelinergic system has been described to promote embryonic and tumor angiogenesis [19,9,43]. Growing evidences of apelin/APJ involvement in embryonic events currently extend beyond vascular development, into ocular [20] and heart development $[42,49,14]$.

Interesting findings regarding the apelinergic system clearly reveal that the lung is one of the organs with strongest expression of both ligand and receptor. Furthermore, emerging evidences of this system's implications in embryonic development prompt the speculation that there might be an underlying role in embryonic lung development. However, the expression profile of this pair of proteins and their functional role during normal lung development is hitherto unknown. So far, the effects of apelin on lung development have been described only in one study that reports attenuation of lung injury in neonatal rats exposed to prolonged hyperoxia [47]. Therefore we proposed to investigate the apelin-APJ system during fetal lung development. In this report a thorough characterization of both components of the apelinergic system by immunohistochemistry and Western blot in several stages of fetal lung development is provided. We further investigated the role of this system in branching morphogenesis of the lung and the intracellular effectors implicated.

\section{Materials and methods}

\subsection{Animal model and experimental design}

Animal experiments were performed according to the Portuguese law for animal welfare. Animals were housed in an accredited mouse house and treated as specified in the "Guide for the Care and Use of Laboratory Animals published by the US National Institutes of Health's (National Institutes of Health Publication No. 85-23, revised 1996). Sprague-Dawley female rats (225 g, Charles-River, Spain) were maintained in appropriate cages under controlled conditions, fed with commercial solid food and after mating they were checked for vaginal plug. The average pregnancy length in normal adult rat is of 21.5 days and the day of plugging was defined as gestational day 0.5 for time dating purposes. Pregnancies were confirmed by regular weightings. At different time-points $[13.5,15.5,17.5,19.5$, and 21.5 days postconception $(\mathrm{dpc})$ ], pregnant female rats were sacrificed by rapid decapitation and fetuses were harvested by cesarean section. Fetuses were also sacrificed by decapitation, fetal and adult lungs were excised, processed and collected for immunohistochemistry (IHC) or Western blot analysis. Regarding lung explant cultures, fetuses were harvested at $13.5 \mathrm{dpc}$ and their lungs were dissected, cultured in vitro for four days and then collected for Western blot analysis.

\subsection{Western blot analysis}

Protein lysates of 13.5 and $15.5 \mathrm{dpc}$ excised lungs and protein lysates of pooled lung explants were obtained by homogenization of the fetal tissue with a pellet pestle motor (Kontes, USA) on ice; as for lungs of later gestational ages and adult samples a mini bead beater (Biospek Products Inc., USA) was used for homogenization. Different pooled lung samples for each gestational age and also the adult were used and three independent experiments were performed. Proteins were obtained according to Kling et al. [24]. Whole protein concentration was quantified by the Bradford method [3]. Either twenty-five or ten micrograms of protein were loaded onto $12.5 \%$ or $10 \%$ acrylamide minigels under denaturing and reducing conditions, electrophoresed for approximately $2 \mathrm{~h}$ at
$100 \mathrm{~V}$ at room temperature and then transferred onto nitrocellulose membranes (Hybond ${ }^{\mathrm{TM}}$-C Extra GE Healthcare Life Sciences, UK) in a wet transfer system for $1 \mathrm{~h}$. Blots were probed with antibodies to apelin [1:500; Apelin (FL-77), Santa Cruz Biotechnology Inc., USA], apelin receptor [1:500; APLNR (H-300), Santa Cruz Biotechnology Inc.] and non-phosphorylated and phosphorylated forms of p38, p44/42 (ERK1/2) and JNK (1:1000; Cell Signaling Technology Inc., USA) according to the manufacturer's instructions. For loading control, blots were reprobed with $\beta$-tubulin antibody (1:150000 Abcam, UK), which were previously incubated with EzWay ${ }^{\mathrm{TM}}$ Antibody Erasing Buffer (Komabiotech Inc., Korea) for primary and secondary antibody removal. Afterwards blots were incubated with a secondary horseradish peroxidase conjugate and developed with Super Signal West Femto Substrate (Pierce Biotechnology Inc., USA). The chemiluminescent signal was captured using a Chemidoc XRS (BioRad, USA) apparatus and subsequent densitometric analysis of nonsaturating bands was performed using Quantity One software (BioRad).

\subsection{Immunohistochemistry}

Immunostaining was performed on paraformaldehyde-fixed and paraffin-embedded excised lungs and embryos. Five micrometers sections were placed onto glass microscope slides. Primary antibodies for apelin [1:50; Apelin (M-77), Santa Cruz Biotechology Inc.] and apelin receptor (APJ, Abcam Inc.) were used. Tissue sections were deparaffinized in xylene and rehydrated in ethanol, boiled in $10 \mathrm{mM}$ citrate buffer for antigen retrieval and cooled down at room temperature. Incubation with the primary antibody occurred at $4{ }^{\circ} \mathrm{C}$ overnight. Negative control reactions included omission of the primary antibody and immunoreactive apelin and APJ staining were not observed in these cases. Sections were incubated with a labeled streptavidin-biotin immunoenzymatic antigen detection system (UltraVision Large Volume Detection System Anti-Polyvalent, Horseradish Peroxidase, Lab Vision Corporation, USA) according to manufacturer's instructions. For visualization of the immune reaction, a diaminobenzidine tetrahydrochloride solution (Dako, Denmark) was used. Sections were finally counterstained with hematoxylin. The slides were observed and photographed with Olympus BX61 microscope (Olympus, Japan). The pictures presented are representative of six animals $(N=6)$, twelve samples were examined for each gestational age as well as the adult and three independent experiments were performed.

\subsection{Fetal lung explant cultures}

Lungs were removed from $13.5 \mathrm{dpc}$ embryos, harvested and dissected under a stereomicroscope (SZX16, Olympus). These were transferred to porous membranes (Isopore ${ }^{\mathrm{TM}}$ membrane filters, Millipore, USA) previously presoaked in DMEM (Invitrogen, UK) and incubated in twenty-four-well culture plates (Nunc, Denmark). Three explants per membrane were positioned in a well-separated arrangement and these floating cultures were incubated at an airmedium interface in a chemically defined medium containing 50\% DMEM, 50\% F-12 nutrient mixture (Invitrogen) and supplemented with $100 \mathrm{mg} / \mathrm{mL}$ penicillin, 100 units/mL streptomycin (Invitrogen), $0.25 \mathrm{mg} / \mathrm{mL}$ ascorbic acid (Sigma-Aldrich, USA) and 10\% FCS (Invitrogen). Fetal lung explants were incubated in a $5 \% \mathrm{CO}_{2}$ incubator at $37^{\circ} \mathrm{C}$ for $96 \mathrm{~h}$, and the medium was replaced every $48 \mathrm{~h}$. Cultures were supplemented daily with several doses of (Pyr1)Apelin-13 (Bachem, Switzerland) ranging from $10^{-11}$ to $10^{-5} \mathrm{M}$. 


\subsection{Morphometric analysis}

Branching morphogenesis was monitored daily by photographing the explants using a stereomicroscope equipped with a camera (DP71, Olympus). At day $0\left(\mathrm{D}_{0}: 0 \mathrm{~h}\right)$ and day $4\left(\mathrm{D}_{4}: 96 \mathrm{~h}\right)$ of culture, the total number of peripheral airway buds in all lung explants was determined. Also, epithelial perimeter, epithelial area and total explant area were measured using Image J image processing and analysis software (version 1.44, USA). For all experimental conditions, the results of the above mentioned morphometric parameters were expressed as $D_{4} / D_{0}$ ratio.

\subsection{Statistical analysis}

All quantitative data are presented as mean \pm SEM. Statistical analysis was performed by one-way ANOVA and subsequently Student-Newman-Keuls test was used for post-test analysis, using SigmaStat 3.5 (Systat Software Inc., USA). Statistical significance was set at $p<0.05$.

\section{Results}

\subsection{Apelin and APJ protein expression profile during rat lung development}

This study aimed to characterize apelin and APJ protein expression profile in the developing rat lung. Western blot and immunohistochemical studies were performed in order to characterize apelin and APJ protein expression levels as well as their localization and distribution during rat pulmonary development. Both proteins were assessed at five prenatal stages, specifically $13.5,15.5,17.5,19.5$ and $21.5 \mathrm{dpc}$ and also in the adult.

\subsubsection{Apelin protein expression profile during rat lung development}

Western blot analysis revealed that apelin is expressed throughout all studied gestational ages in fetal lung as well as in the adult lung (Fig. 1). Apelin expression levels were maximal at the earliest gestational age studied, $13.5 \mathrm{dpc}$, early pseudoglandular stage in lung development, and then declined irregularly toward the end of gestation. Lowest apelin expression levels in prenatal period were detectable at $17.5 \mathrm{dpc}$, late pseudoglandular stage. In the adult rat lung, apelin expression levels decline to a statistically significant remarkable minimum.

Regarding immunohistochemical studies (Fig. 2), at the earliest gestational age studied, $13.5 \mathrm{dpc}$, apelin-positive cells are detected in both major tissues shaping the embryonic lung, epithelium and mesenchyme, in an extensive fashion (Fig. $2 \mathrm{~A}$ and $\mathrm{A}^{\prime}$ ). At $15.5 \mathrm{dpc}$, immunostaining was observed in the epithelium of the developing airways as well as in the mesenchyme surrounding the airways and also in the endothelium (Fig. 2B and $\mathrm{B}^{\prime}$ ). At late pseudoglandular stage, $17.5 \mathrm{dpc}$, apelin immunostaining is observed in the whole organ, more intensely in the epithelial lining of the airways, also in surrounding mesenchyme and the vascular endothelium (Fig. 2C and $C^{\prime}$ ). The same expression pattern is detected in canalicular stage of development, $19.5 \mathrm{dpc}$ (Fig. 2D and $\left.\mathrm{D}^{\prime}\right)$. At the later gestational age, $21.5 \mathrm{dpc}$, apelin immunostaining is primarily associated with both bronchial and alveolar epithelium and the vascular endothelium (Fig. $2 \mathrm{E}$ and $\mathrm{E}^{\prime}$ ). The adult rat lung seems to exhibit a dissimilar expression profile from the one observed during gestational stages, bronchial epithelial staining and endothelial expressions are no longer predominant, alveolar epithelium has more significant expression, and there is also positive immunostaining detectable in blood (Fig. $2 \mathrm{~F}$ and $\mathrm{F}^{\prime}$ ).

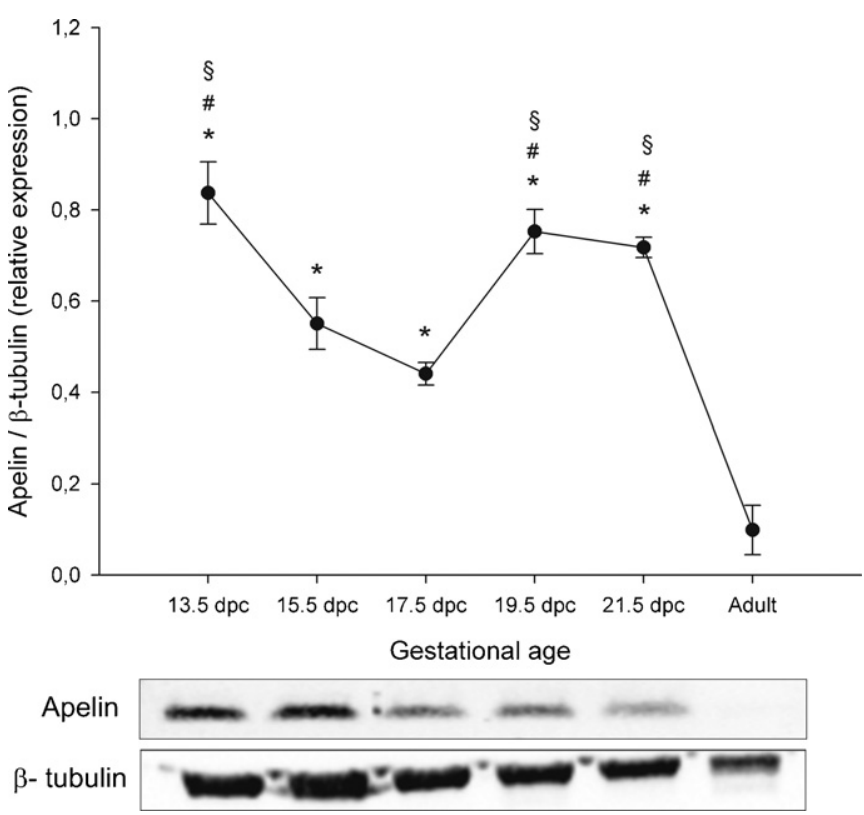

Fig. 1. Protein levels of apelin during fetal rat lung development. Western blot analysis of apelin performed in several gestational ages, from 13.5 to $21.5 \mathrm{dpc}$ and also in the adult. Representative blot examples are shown. All protein levels were normalized to $\beta$-tubulin, which was used as loading control. Densitometric analyses of three blots $(N=3)$ are plotted above the representative blots. Values represent mean \pm SE. $p<0.05:^{*} v s$. adult; \# vs. $17.5 \mathrm{dpc}$; $v s .15 .5 \mathrm{dpc}$.

\subsubsection{APJ protein expression profile during rat lung development}

Western blot analysis of the apelin receptor, APJ, revealed immunoreactivity for specific bands correlating to an expected molecular mass of $\sim 42 \mathrm{kDa}$ for the unglycosylated monomeric receptor as well as bands of higher molecular mass consistent with glycosylated monomeric receptor, $\sim 45 \mathrm{kDa}$. Additional bands consistent in molecular weight to multiples of the monomer were also observed, dimeric receptor species at $90-95 \mathrm{kDa}$, and oligomeric receptor species at $125-135 \mathrm{kDa}$.

Both monomeric and multimeric forms of the apelin receptor were expressed throughout all studied gestational ages in fetal lung as well as in the adult, although exhibiting different expression profiles (Fig. 3). As for the monomeric protein (Fig. 3A), highest expression levels were detected at the earliest gestational age studied, $13.5 \mathrm{dpc}$, decreasing progressively at 15.5 and $17.5 \mathrm{dpc}$, the latter being the stage in which expression level was lowest during the prenatal period. Stages 19.5 and $21.5 \mathrm{dpc}$ show a slight increase in the protein expression levels comparing to $17.5 \mathrm{dpc}$, nonetheless the same progressively decreasing tendency in APJ monomer protein expression during development prevails. Lowest expression levels with statistically significance were detected in the adult rat lung. Regarding the dimeric form of the receptor (Fig. 3B), despite no statistical difference established, adult rat lung has the highest expression level detected. APJ dimeric expression levels throughout all gestational ages studied remained mostly unvarying. Regarding the oligomeric form of the receptor (Fig. 3C), again highest expression levels were detected at the earliest gestational age studied, $13.5 \mathrm{dpc}$, decreasing progressively into the later gestational age. Also for this form of the receptor, lowest expression levels were detected in the adult rat lung.

Considering immunohistochemistry data, at the earliest gestational stages studied, 13.5 and $15.5 \mathrm{dpc}$, apelin receptor immunoreactivity observed has a very similar distribution pattern to that of apelin immunoreactivity (Fig. 4). In the first, there is APJ-positive cells in both epithelial and mesenchymal tissue with comparable intensity. At the latter stage, positivity is detected in the epithelium of the airways, in the mesenchyme surrounding 

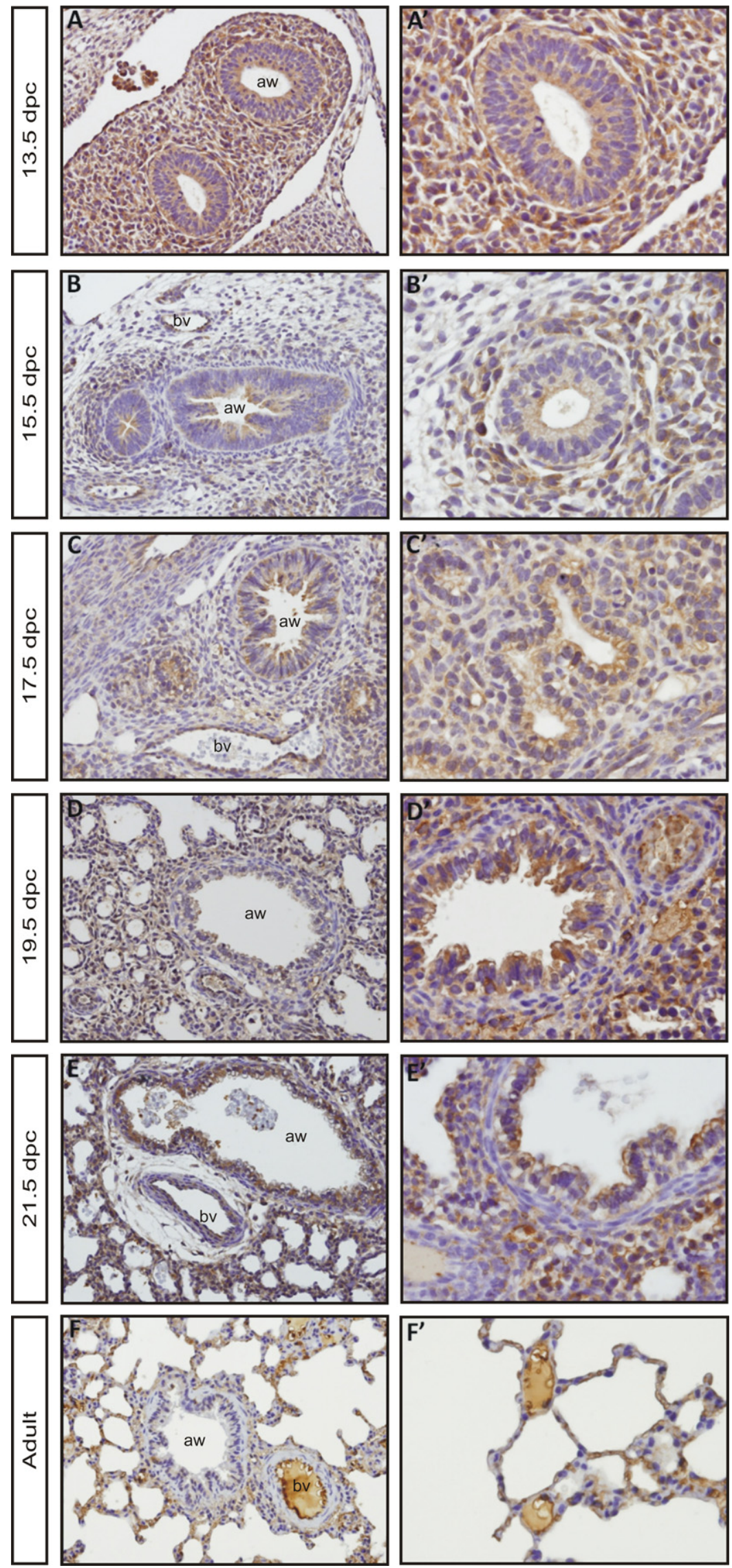

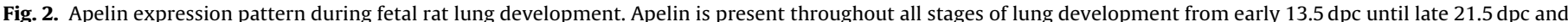

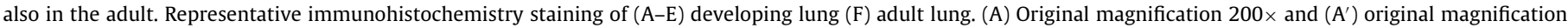
$400 \times$. aw: developing airway and bv: blood vessel. 

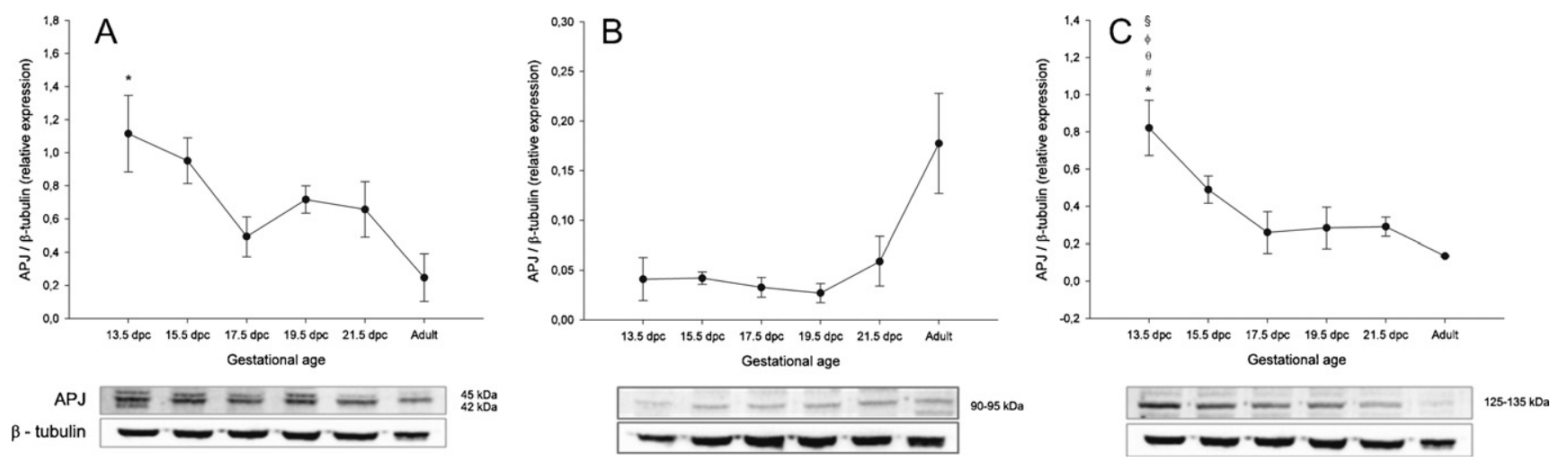

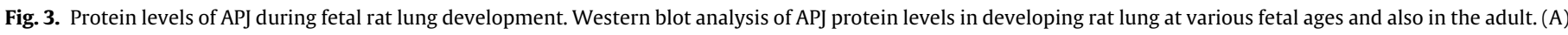

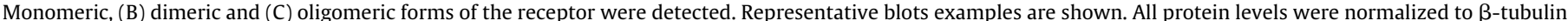

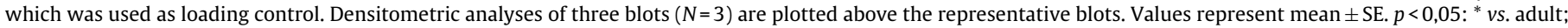
$\#$ vs. $17.5 \mathrm{dpc}, \theta$ vs. $19.5 \mathrm{dpc}, \phi$ vs. $21.5 \mathrm{dpc}$, and § vs. $15.5 \mathrm{dpc}$.

the airways and also in the endothelium (Fig. 4A, B, $\mathrm{A}^{\prime}$ and $\mathrm{B}^{\prime}$ ). At stage $17.5 \mathrm{dpc}$, although there is widespread staining detectable in the organ, it is more intense in the cells of the epithelial lining of the airways and staining is predominantly nuclear, there is also weaker staining in the vascular endothelium and surrounding areas (Fig. $4 C$ and $C^{\prime}$ ). At one of the later gestational ages studied, $19.5 \mathrm{dpc}$, it is also observed a broad distribution of the apelin receptor in the fetal lung, but immunoreactivity is more intense in the airway epithelial cells, in which positivity is detected in the nucleus, the cytoplasm and the membrane (Fig. 4D and $\mathrm{D}^{\prime}$ ). By the end of gestation at $21.5 \mathrm{dpc}$, APJ expression is also related mainly to the bronchial and alveolar epithelium, which is the predominant tissue at this stage, weaker expression is detectable in the endothelial lining of the vasculature (Fig. $4 \mathrm{E}$ and $\mathrm{E}^{\prime}$ ). In the adult rat lung, a weak APJ expression is detectable in the alveoli that comprises most of the respiratory tissue, and also in the endothelial lining of the vessels and the smooth muscle surrounding bronchi and large pulmonary vessels. More intense staining is detected in the nucleus and cytoplasm of the epithelial cells of the respiratory bronchi (Fig. $4 \mathrm{~F}$ and $\mathrm{F}^{\prime}$ ).

\subsection{Effects of apelin supplementation in fetal lung explant cultures}

This study also aimed to elucidate the role of apelin in the developing rat lung. Therefore, apelin supplementation studies were carried out in the fetal lung explant culture model. Normal fetal lung explants were treated with different doses of recombinant (Pyr1)Apelin-13. In Fig. 5, representative examples of normal fetal lung explants treated with increasing Apelin-13 doses are illustrated. Apelin-13 appears to have an inhibitory effect on lung explants growth, except with the lowest dose tested as shown by the morphometric analysis data summarized in Fig. 6. In fetal lung explants, regardless of a slight increase in growth verified in all the morphometric parameters induced by the lowest dose of Apelin-13 tested, no statistical significance against the absence of apelin treatment was observed. In contrast, supplementation with the highest dose, $10^{-5} \mathrm{M}$, significantly reduced whole explant growth when compared with the lowest dose of Apelin-13 tested, demonstrated by both epithelial and external explant area. Also, considering the epithelial perimeter, a significant inhibitory effect is induced by the highest dose tested when compared to lack of supplementation or treatment with low Apelin-13 doses, $10^{-11}$ and $10^{-9} \mathrm{M}$. Considering the number of peripheral airway buds, no significant statistical difference was observed.

\subsection{Apelin supplementation effects on MAPK signaling pathway}

In order to further investigate the underlying molecular mechanisms responsible for the effect of apelin on fetal lung growth, the dose effect of apelin on mitogen-activated protein kinases signaling was determined. Expression levels of p38, JNK1/2, and ERK1/2 were assessed by Western blot in pooled samples $(N=12)$ of Apelin13 treated fetal lung explants. Activation of MAPKs is regulated by phosphorylation, thus both unphosphorylated and phosphorylated protein states were assessed, as shown in Fig. 7. For each protein the ratio of phosphorylated to unphosphorylated form was determined (Fig. 8) revealing that apelin treatment induced a dose dependent decrease in activation of both $\mathrm{p} 38$ and JNK, since increasing doses of apelin gradually reduced phosphorylation of these two MAP kinases (Fig. 8A and B). In contrast, the highest dose of apelin tested induced activation of ERK $1 / 2$, whereas supplementation with lower doses of apelin reduced phosphorylation when compared with absence of apelin treatment (Fig. 8C).

\section{Discussion}

This study analyses for the first time, fetal pulmonary protein expression profile of both components of the apelinergic system, apelin and APJ, by Western blot and immunohistochemistry, parallel comparison with the adult profile is also presented. Additionally, in vitro apelin supplementation studies were performed in fetal lung explants in order to elucidate its putative role in branching morphogenesis and we further investigated MAPK signaling contribution to the effects of apelin on fetal lung growth. We demonstrated that both components of the apelinergic system are constitutively expressed in the lung during all studied gestational ages and also in the adult. Western blot analysis yields important data regarding the characterization of apelin and APJ in lung development. Apelin immunoreactivity has been previously detected as a band of approximately $16 \mathrm{kDa}$ in different tissue lysates [28], as it is observed in our sample lysates. In fetal lung, apelin protein expression levels have a maximum at an early stage in development $(13.5 \mathrm{dpc})$, in all the other gestational ages studied expression levels are lower, and its minimum is reached in the adult tissue. This fact, points toward, possibly, a more relevant role of this peptide during early stages of lung development. Regarding apelin receptor, Western blot analysis made it possible to observe that this receptor exits in a variety of presentations in the pulmonary tissue, which include post-translational modifications and multimeric arrangements. At lower molecular weight, both unglycolysated and glycolysated forms (42 and 

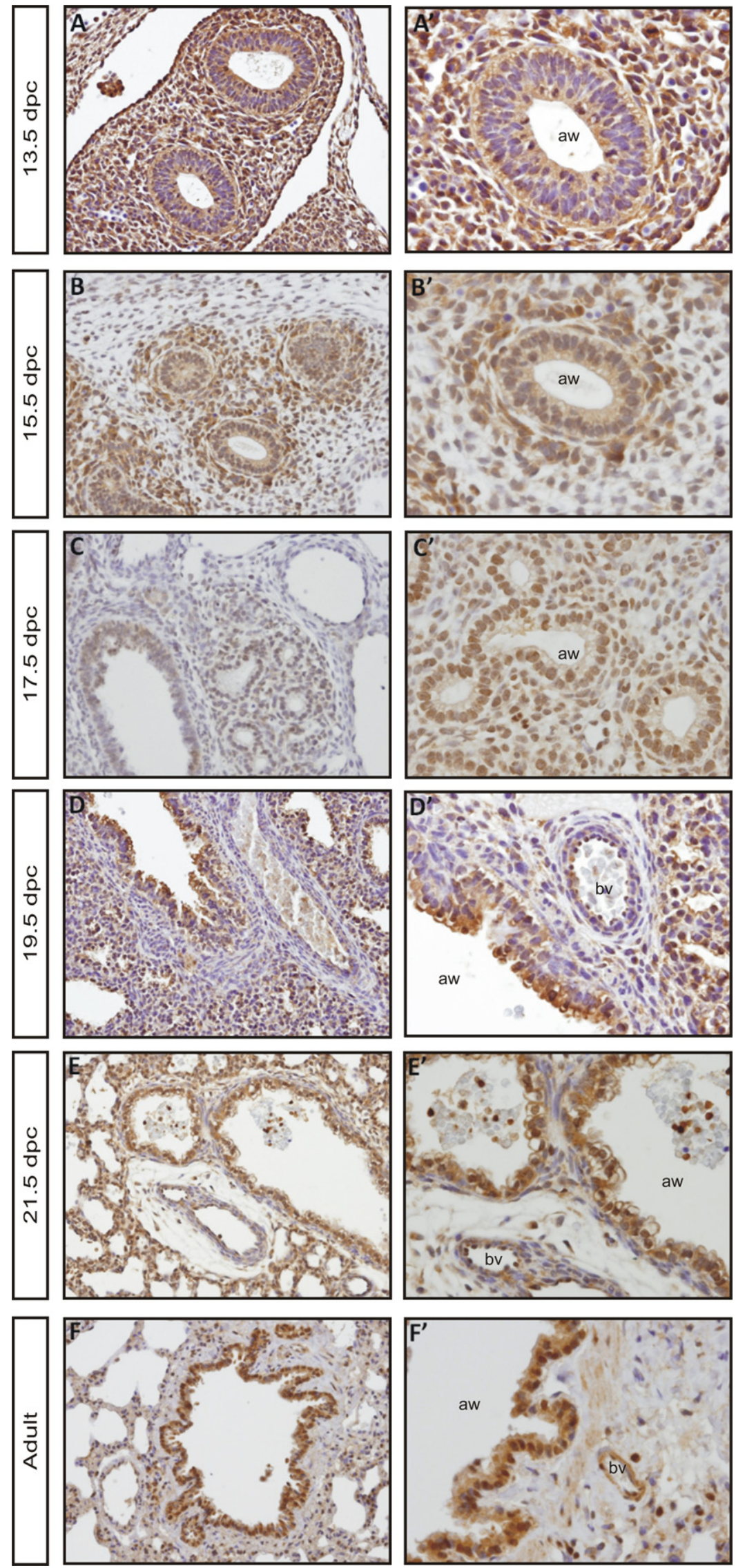

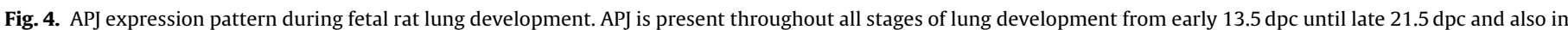

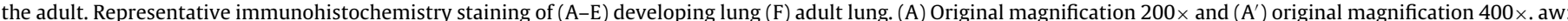
developing airway and bv: blood vessel. 


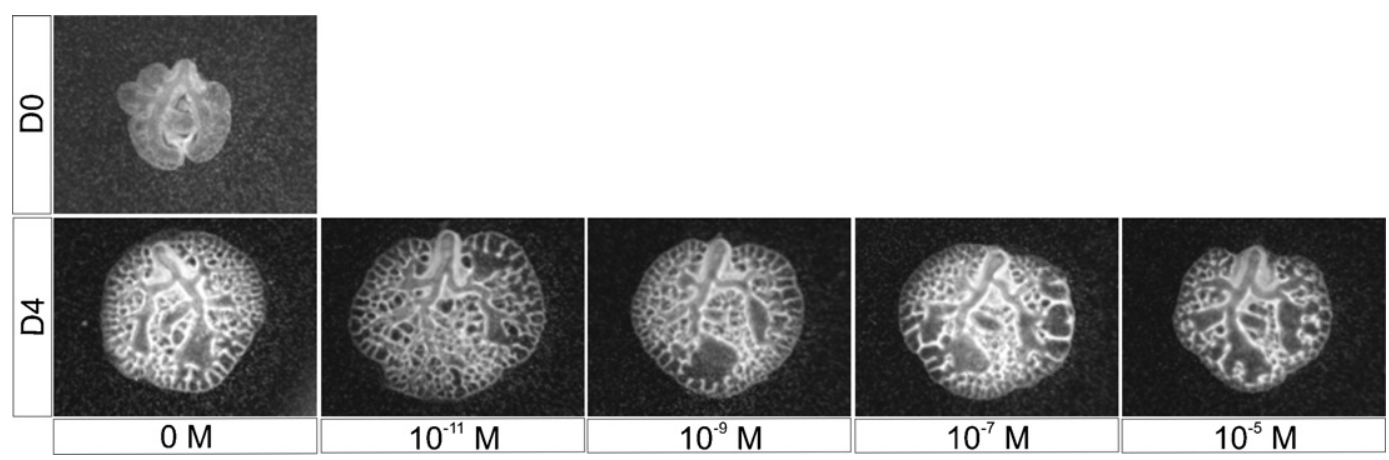

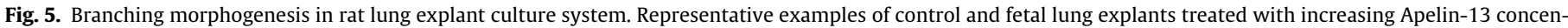

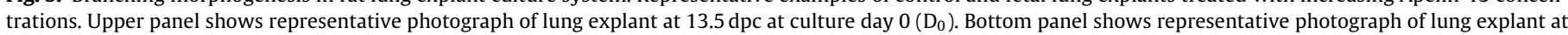
$13.5 \mathrm{dpc}$, treated with several Apelin-13 concentrations, at day 4 in culture $\left(\mathrm{D}_{4}\right)$. Original magnification $40 \times$.

$45 \mathrm{kDa}$ ) of the monomeric receptor species are present in all studied gestational ages. At higher molecular weights, corresponding to multiples of the monomer, dimeric ( $\sim 90-95 \mathrm{kDa})$ and oligomeric ( $\sim 125-135 \mathrm{kDa}$ ) receptor species were observed. According to the literature many GPCRs, besides the monomeric form, are expressed as dimers and oligomers, but it is also common for GPCR to appear as homo- and hetero-dimers and oligomers $[5,41,33]$. Whether APJ multimeric species observed in the fetal and adult pulmonary tissues in our study correspond to homo- or hetero-multiples of the receptor is still not known and could only be clarified by further studies. Nonetheless, it was already demonstrated in vitro that APJ can form heterodimers with angiotensin II AT1 receptor [7]. Moreover immunohistochemical studies performed in our lab revealed that all renin-angiotensin system components, including AT1 and
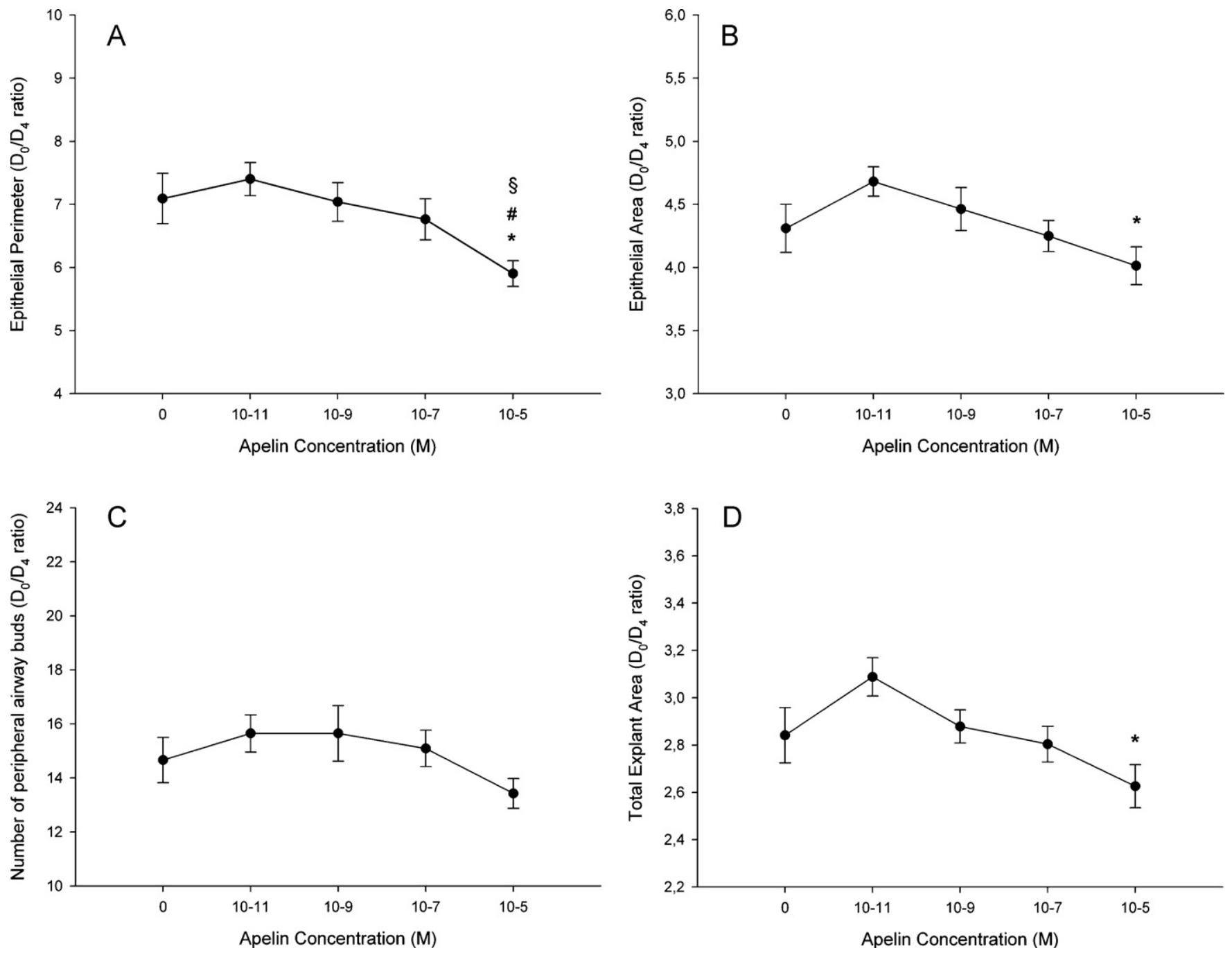

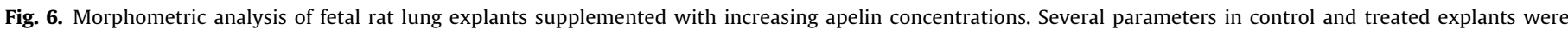

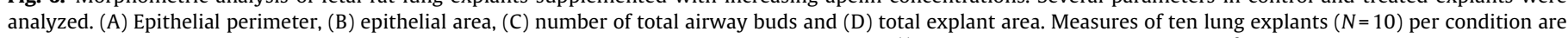
plotted above. Results are expressed as $D_{4} / D_{0}$ ratio. Values represent mean \pm SE. $p<0,05:^{*} v s .10^{-11} \mathrm{M}$, \# vs. $0 \mathrm{M}$ (control), and $\S$ vs. $10^{-9} \mathrm{M}$. 

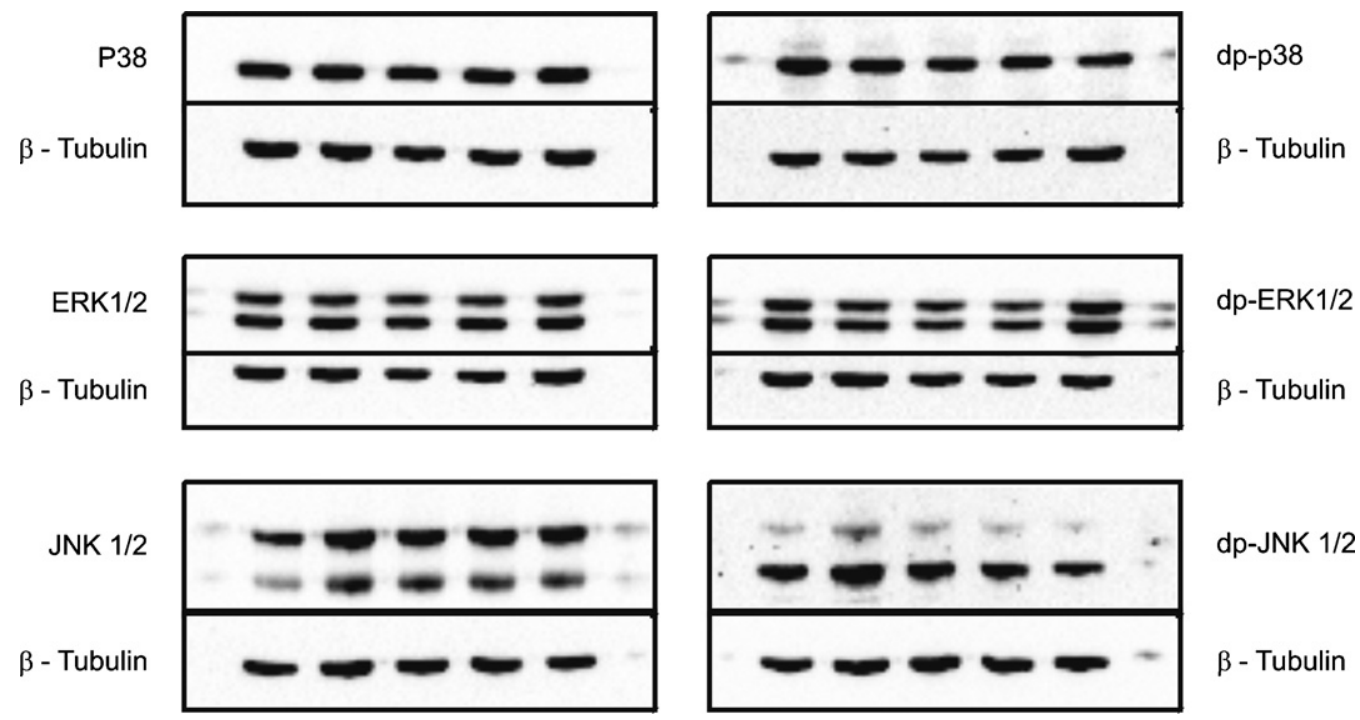

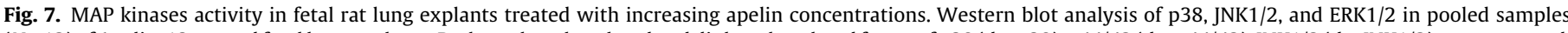

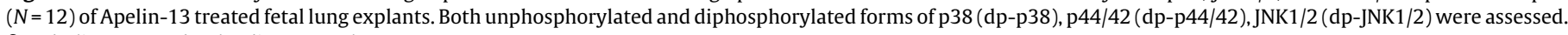
$\beta$-Tubulin was used as loading control.

AT2 receptors, are expressed in fetal lung throughout the gestational ages also characterized in this study [36].

The immunohistochemical studies were performed to further characterize the presence of apelin and APJ in the pulmonary tissue. These studies allow us to conclude that apelin lung expression is mainly related to both airways and vasculature during the developmental stages studied. Apelin immunostaining was detected in the airways lining, either in bronchial epithelium throughout gestation, as well as in the alveolar epithelium as it gradually appeared. Regarding the vasculature, positive immunostaining was detected in the endothelial lining of the blood vessels from $15.5 \mathrm{dpc}$ to $21.5 \mathrm{dpc}$. Whereas in the adult rat lung, apelin presence is strongest in the alveolar epithelium, less intense in the bronchial epithelium and nearly absent in the vasculature. Interestingly there is apelin immunoreactivity in the content of the pulmonary blood vessels which is not associated with blood cells, which may possibly be in accordance with previous reports that detected plasma levels of apelin in humans and consequently showed that apelin is a circulating peptide $[6,13,16]$. Few studies report apelin localization in pulmonary tissue by immunohistochemistry, showing intense staining in the respiratory epithelium and smooth muscle in normal adult human tissues [10], and also presence in bronchial and alveolar epithelial cells in neonatal rat lung [47].

Immunohistochemistry for the receptor protein revealed that APJ localization is predominantly associated with the pulmonary airways in the developmental stages studied. Most striking is the distribution of the receptor within the bronchial epithelium, where APJ can be detected either in the cytoplasm, the membrane and also the nucleus of these epithelial cells, nuclear localization of the receptor appears as early as $15.5 \mathrm{dpc}$ and is detectable in all subsequent stages. Apelin receptor nuclear localization is therefore indicative of nuclear translocation of APJ occurring probably only in certain cell populations within pulmonary tissue. To date, there have been many reports of the intercellular localization and trafficking of GPCRs, although GPCRs are best known as cell surface mediators of signal transduction there is an increasing number of reports of GPCRs capable of nuclear translocation [15]. Thus far, besides our findings in pulmonary tissue there is only one report showing that apelin receptor exhibits nuclear localization occurring in brain tissue [27]. This nuclear localization of apelin receptor points out to previously unforeseen functions of this receptor as a modulator of nuclear transcription that are worth investigating, in addition to the well-established role of this receptor family at the cell surface. In the adult rat lung, APJ immunostaining is most intense in the bronchial epithelium and respiratory epithelium where it is observed that expression is cytoplasmatic, membranous and nuclear. Additionally, lower intensity immunostaining was detected in the alveolar epithelium, the endothelium and also the smooth muscle around the bronchi. These results match previous findings of immunoreactivity in human lung tissue that showed that APJ is expressed in endothelial cells and smooth muscle of small pulmonary vessels, and also demonstrated receptor
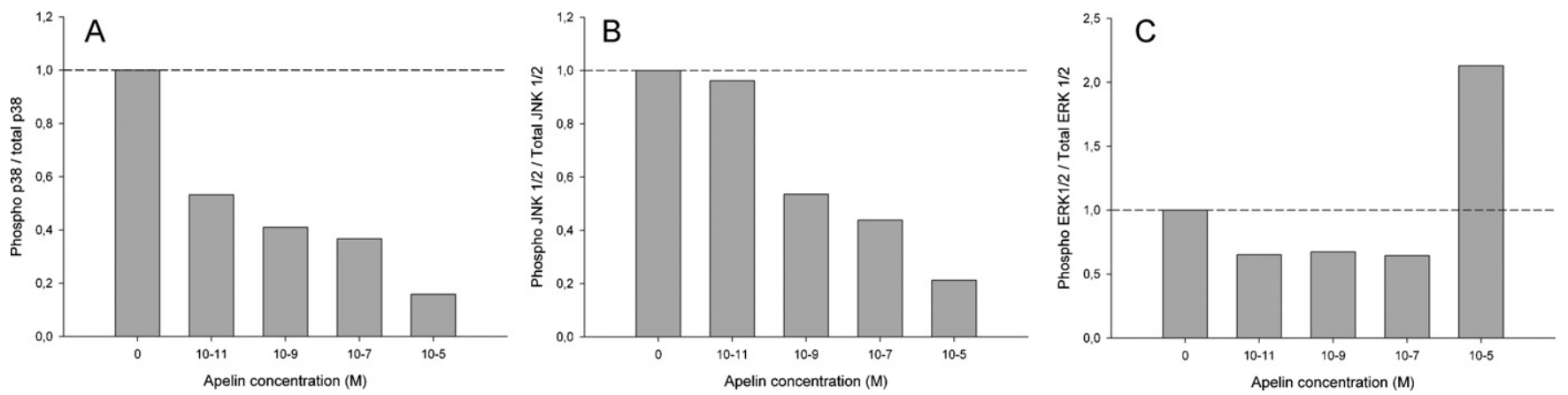

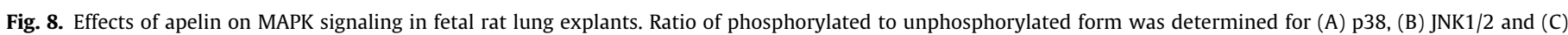
ERK1/2. All protein levels were normalized to $\beta$-tubulin, which was used as loading control. Densitometric analysis results are presented as arbitrary units. 
immunoreactivity in bronchial epithelial cells as well as in the endothelium and in smooth muscle cells in rat tissue [23]. The fetal lung displays the necessary machinery for apelin/APJ signaling, and the epithelium might act as both the physiological source of apelin and also as a target tissue for apelin to exert its physiological action during fetal lung development.

Our findings on the presence and significant features regarding the characterization of both components of this peptidergic system on the pulmonary tissue during development led us to hypothesize that it might have an underlying role in lung branching morphogenesis. Hence, in order to begin exploring this assumption, in vitro apelin supplementation studies were performed in the fetal lung explant culture model. The morphometric analysis on the fetal lung explants exposed to increasing doses of apelin in culture, showed a steady tendency for an inhibition of lung explant growth, especially significant in the highest dose tested. It is well known that negative molecular mediators are essential in pulmonary development given that there is a strict dependence on the balance between inductive and suppressive mechanisms occurring in this process. Therefore, orderly branching morphogenesis also depends on multiple antagonists [34,48]. Interestingly, it was recently demonstrated in the same in vitro culture model, that angiotensin II (Ang II) supplementation induced an increase in lung explants growth [36]. Most striking about these findings is its straight association with an emerging key feature of the apelin/APJ system, its interaction with the renin-angiotensin system. Currently the relation between these two systems is described to be a direct interaction of the two systems at both molecular and transcriptional levels to mediate opposing physiological actions [2]. APJ and AT1 receptor-receptor interactions are presently accounted as the potential molecular mechanism of crosstalk responsible for this reciprocal counter-regulation between apelin and Ang II pathways, since it was observed in vitro that the respective receptors can form heterodimers and that this heterodimerisation influences downstream signaling [7]. Our observations on fetal lung growth are in accordance with others reporting that the proposed effects of the apelin-APJ system are opposite to those of the Ang II-AT1 receptor pathway $[7,44]$.

On one hand, coupling of apelin to its receptor APJ has been reported to activate MAP kinases signaling pathways $[1,28]$. On the other hand, MAPK were shown to be important signaling pathway for murine lung development through regulation of branching morphogenesis, epithelial cell proliferation, cell survival and differentiation [24]. Hence, evaluating MAPK signaling in the context of elucidating apelin's effects on fetal lung growth was regarded pertinent. Our results clearly show dose dependent decrease in activation of both p38 and JNK induced by apelin treatment as well as activation of ERK1/2 induced by the highest dose of apelin tested. In previous studies enhanced fetal lung branching was associated with stimulation of p38 phosphorylation [37] likewise, lung growth inhibition induced by AT1 antagonist was reported to be mediated by a decrease of p38 and JNK phosphorylation [36]. Therefore, apelin supplementation inhibitory effect on lung growth is most likely mediated by decreased p38 and JNK phosphorylation. It is common for MAP kinases to share substrates and to integrate signals from individual cascades not only during the early signal transduction events and within the kinase cascades, but also at the level of substrate phosphorylation [8]. In view of this, it is only reasonable to assume that these apparent discrepancies in the expression of $\mathrm{p} 38$, JNK and ERK1/2 might reflect cross-cascade interactions taking place in order to restore intracellular signaling balance.

In conclusion, the data here presented provides the first evidence of the presence of an apelinergic system operating in the developing lung. Our results also demonstrated that the apelin receptor exhibits nuclear localization in lung epithelial cells, which unveils an important and novel feature of this system that suggest unanticipated functions for this G-protein coupled receptor in nuclear transcription regulation. Moreover, it is also demonstrated that apelin's inhibitory effect on fetal lung growth is associated with inhibition of $\mathrm{p} 38$ and JNK signaling pathways. We have further characterized this novel peptidergic system in the developing lung.

\section{Acknowledgement}

The authors wish to thank Miguel Carvalho and Luís Martins for skillful assistance in animal euthanasia.

\section{References}

[1] Bai B, Tang J, Liu H, Chen J, Li Y, Song W. Apelin-13 induces ERK1/2 but not p38 MAPK activation through coupling of the human apelin receptor to the $\mathrm{Gi} 2$ pathway. Acta Biochim Biophys Sin 2008;40(4):311-8.

[2] Barnes G, Japp AG, Newby DE. Translational promise of apelin-APJ system. Heart 2010;96:1011-6.

[3] Bradford MM. Rapid and sensitive method for the quantitation of microgram quantities of protein utilizing the principle of protein-dye binding. Anal Biochem 1976;72:248-54.

[4] Castan-Laurell I, Dray C, Attané C, Duparc T, Knauf C, Valet P. Apelin, diabetes, and obesity. Endocrine 2011;40(1):1-9.

[5] Ciruela F, Vilardaga JP, Fernández-Dueñas V. Lighting up multiprotein complexes: lessons from GPCR oligomerization. Trends Biotechnol 2010;28(8):407-15.

[6] Cheng X, Cheng XS, Pang CC. Venous dilator effect of apelin, an endogenous peptide ligand for the orphan APJ receptor, in conscious rats. Eur J Pharmacol 2003;470:171-5.

[7] Chun HJ, Ali ZA, Kojima Y, Kundu RK, Sheikh AY, Agrawal R, et al. Apelin signaling antagonizes Ang II effects in mouse models of atherosclerosis. J Clin Invest 2008:118(10):3343-54.

[8] Cobb MH. MAP kinase pathways. Prog Biophys Mol Biol 1999;71(3-4):479-500.

[9] Cox CM, D’Agostino SL, Miller MK, Heimark RL, Krieg PA. Apelin, the ligand for the endothelial G-protein-coupled receptor, APJ, is a potent angiogenic factor required for normal vascular development of the frog embryo. Dev Biol 2006;296(1):177-89.

[10] De Falco M, De Luca L, Onori N, Cavallotti I, Artigiano F, Esposito V, et al. Apelin expression in normal human tissues. In Vivo 2002;16(5):333-6.

[11] Drews J. Drug discovery: a historical perspective. Science 2000;287:1960-4.

[12] Falcão-Pires I, Ladeiras-Lopes R, Leite-Moreira AF. The apelinergic system: a promising therapeutic target. Expert Opin Ther Targets 2010;14(5):1-13.

[13] Földes G, Horkay F, Szokodi I, Vuolteenaho O, Ilves M, Lindstedt KA, et al. Circulating and cardiac levels of apelin, the novel ligand of the orphan receptor APJ, in patients with heart failure. Biochem Biophys Res Commun 2003;308(3):480-5.

[14] Gao LR, Zhang NK, Bai J, Ding QA, Wang ZG, Zhu ZM, et al. The apelin-AP pathway exists in cardiomyogenic cells derived from mesenchymal stem cells in vitro and in vivo. Cell Transplant 2010;19:949-58.

[15] Gobeil F, Fortier A, Zhu T, Bossolasco M, Leduc M, Grandbois M, et al. G-proteincoupled receptors signalling at the cell nucleus: an emerging paradigm. Can J Physiol Pharmacol 2006;84(3-4):287-97.

[16] Goetze JP, Rehfeld JF, Carlsen J, Videbaek R, Andersen CB, Boesgaard S, et al. Apelin: a new plasma marker of cardiopulmonary disease. Regul Pept 2006;133(1-3):134-8.

[17] Habata Y, Fujii R, Hosoya M, Fukusumi S, Kawamata Y, Hinuma S, et al. Apelin, the natural ligand of the orphan receptor APJ, is abundantly secreted in the colostrum. Biochim Biophys Acta 1999;1452:25-35.

[18] Hosoya M, Kawamata Y, Fukusumi S, Fujii R, Habata Y, Hinuma S, et al. Molecular and functional characteristics of APJ: tissue distribution of mRNA and interaction with the endogenous ligand apelin. J Biol Chem 2000;275:21061-7.

[19] Kälin RE, Kretz MP, Meyer AM, Kispert A, Heppner FL, Brändli AW. Paracrine and autocrine mechanisms of apelin signaling govern embryonic and tumor angiogenesis. Dev Biol 2007;305:599-614.

[20] Kasai A, Shintani N, Kato H, Matsuda S, Gomi F, Haba R, et al. Retardation of retinal vascular development in apelin-deficient mice. Arterioscler Thromb Vasc Biol 2008;28:1717-22.

[21] Kawamata Y, Habata Y, Fukusumi S, Hosoya M, Fujii R, Hinuma S, et al. Molecular properties of apelin: tissue distribution and receptor binding. Biochim Biophys Acta 2001;1538:162-71.

[22] Kleinz MJ, Davenport AP. Emerging roles of apelin in biology and medicine. Pharmacol Ther 2005;107(2):198-211.

[23] Kleinz MJ, Skepper JN, Davenport AP. Immunocytochemical localisation of the apelin receptor, APJ, to human cardiomyocytes, vascular smooth muscle and endothelial cells. Regul Pept 2005;126(3):233-40.

[24] Kling DE, Lorenzo HK, Trbovich AM, Kinane TB, Donahoe PK, Schnitzer JJ. Preand postnatal lung development, maturation, and plasticity: MEK-1/2 inhibition reduces branching morphogenesis and causes mesenchymal cell apoptosis in fetal rat lungs. Am J Physiol Lung Cell Mol Physiol 2002;282:L370-8.

[25] Lee DK, Cheng R, Nguyen T, Fan T, Kariyawasam AP, Liu Y, et al. Characterization of apelin, the ligand for the APJ receptor. J Neurochem 2000;74:34-41. 
[26] Lee DK, George SR, O'Dowd BF. Unravelling the roles of the apelin system: prospective therapeutic applications in heart failure and obesity. Trends Pharmacol Sci 2006;27(4):190-4.

[27] Lee DK, Lanca AJ, Cheng R, Nguyen T, Ji XD, Gobeil Jr F, et al. Agonist-independent nuclear localization of the Apelin, angiotensin AT1, and bradykinin B2 receptors. J Biol Chem 2004;279(9):7901-8.

[28] Lee DK, Saldivia VR, Nguyen T, Cheng R, George SR, O‘Dowd BF. Modification of the terminal residue of apelin-13 antagonizes its hypotensive action. Endocrinology 2005;146(1):231-6.

[29] Liu C, Su T, Li F, Li L, Qin X, Pan W, et al. PI3K/Akt signaling transduction pathway is involved in rat vascular smooth muscle cell proliferation induced by apelin13. Acta Biochim Biophys Sin 2010;42(6):396-402.

[30] Masri B, Lahlou H, Mazarguil H, Knibiehler B, Audigier Y. Apelin (65-77) activates extracellular signal-regulated kinases via a PTX-sensitive G protein. Biochem Biophys Res Commun 2002;290(1):539-45.

[31] Masri B, Morin N, Cornu M, Knibiehler B, Audigier Y. Apelin (65-77) activates p70 S6 kinase and is mitogenic for umbilical endothelial cells. FASEB 2004;18(15):1909-11.

[32] Medhurst AD, Jennings CA, Robbins MJ, Davis RP, Ellis C, Winborn KY, et al. Pharmacological and immunohistochemical characterization of the APJ receptor and its endogenous ligand apelin. J Neurochem 2003;84(5):1162-72.

[33] Milligan G. G protein-coupled receptor dimerization: function and ligand pharmacology. Mol Pharmacol 2004;66(1):1-7.

[34] Morrisey EE, Hogan BL. Preparing for the first breath: genetic and cellular mechanisms in lung development. Dev Cell 2010;18:8-23.

[35] Neves SR, Ram PT, Iyengar R. G protein pathways. Science 2002;296(5573):1636-9.

[36] Nogueira-Silva C, Carvalho-Dias E, Piairo P, Nunes S, Baptista MJ, Moura RS, et al. Local Fetal lung renin-angiotensin system as a target to treat congenital diaphragmatic hernia. Personal communication.

[37] Nogueira-Silva C, Santos M, Baptista MJ, Moura RS, Correia-Pinto J. IL-6 is constitutively expressed during lung morphogenesis and enhances fetal lung explant branching. Pediatr Res 2006;60:530-6.
[38] O'Dowd BF, Heiber M, Chan A, Heng HHQ Tsui LC, Kennedy JL, et al. A human gene that shows identity with the gene encoding the angiotensin receptor is located on chromosome 11. Gene 1993;136:355-60.

[39] Quazi R, Palaniswamy C, Frishman WH. The emerging role of apelin in cardiovascular disease and health. Cardiol Ver 2009;17:283-6.

[40] Reaux A, De Mota N, Skultetyova I, Lenkei Z, El Messari S, Gallatz K, et al. Physiological role of a novel neuropeptide, apelin, and its receptor in the rat brain. J Neurochem 2001;77:1085-96.

[41] Rovira X, Pin JP, Giraldo J. The asymmetric/symmetric activation of GPCR dimers as a possible mechanistic rationale for multiple signalling pathways. Trends Pharmacol Sci 2010;31(1):15-21.

[42] Scott IC, Masri B, D'Amico LA, Jin SW, Jungblut B, Wehman AM, et al. The C protein-coupled receptor agtrl1b regulates early development of myocardial progenitors. Dev Cell 2007;12(3):403-13.

[43] Sorli SC, Le Gonidec S, Knibiehler B, Audigier Y. Apelin is a potent activator of tumour neoangiogenesis. Oncogene 2007;26(55):7692-9.

[44] Sun X, Iida S, Yoshikawa A, Senbonmatsu R, Imanaka K, Maruyama K, et al. Nonactivated APJ suppresses the angiotensin II type 1 receptor, whereas apelinactivated APJ acts conversely. Hypertens Res 2011;34(6):701-6.

[45] Tatemoto K, Hosoya M, Habata Y, Fujii R, Kakegawa T, Zou MX, et al. Isolation and characterization of a novel endogenous peptide ligand for the human AP receptor. Biochem Biophys Res Commun 1998;251:471-6.

[46] Tatemoto K, Takayama K, Zou MX, Kumaki I, Zhang W, Kumano K, et al. The novel peptide apelin lowers blood pressure via a nitric oxide-dependent mechanism. Regul Pept 2001;99(2-3):87-92.

[47] Visser YP, Walther FJ, Laghmani el H, Laarse A, Wagenaar GT. Apelin attenuates hyperoxic lung and heart injury in neonatal rats. Am J Respir Crit Care Med 2010;182(10):1239-50.

[48] Warburton D, Schwarz M, Tefft D, Flores-Delgado G, Anderson KD, Cardoso WV. The molecular basis of lung morphogenesis. Mech Dev 2000;92(1):55-81.

[49] Zeng XX, Wilm TP, Sepich DS, Solnica-Krezel L. Apelin and its receptor control heart field formation during zebrafish gastrulation. Dev Cell 2007;12(3):391-402. 